\title{
Synthesis of 3-methoxy-6-phenyl-6, 6a-dihydro-[1] benzopyrano- [3, 4-b] [1] benzopyran.
}

\author{
Aimé-Jhustelin Abogo Mebale ${ }^{1}$, Alain Maggiani ${ }^{2}$, André Samat ${ }^{2}$ \\ ${ }^{I}$ Ecole Normale Supérieure, Laboratoire Pluridisciplinaire des Sciences (LAPLUS), \\ B.P. 17009 Libreville, Gabon. \\ ${ }^{2}$ CINaM, CNRS UMR 7325, Aix-Marseille Université Faculté des Sciences de Luminy, 163 Avenue de \\ Luminy, Case 901 -13288 Marseille Cedex 9 France.
}

\begin{abstract}
The 3-methoxy-6-phenyl-6,6a-dihydro-[1]benzopyrano-[3,4-b][1]benzopyran has been synthesised according to eight-steps synthesis. The target compound was obtained using a synthetic route based on two key cyclisations steps including the platinum chlorides-catalysed of alkynones and intramolecular oxo-Michael addition.
\end{abstract}

Keywords - 3-methoxy-6-phenyl-6,6a-dihydro-[1]benzopyrano-[3,4-b][1]benzopyran, photochromic system, oxo-Michael addition.

\section{INTRODUCTION}

Photochromic compounds are very interesting substances able to undergo a photoinduced change of color. Many studies have been done since 1950, particularly concerning benzo and naphthopyrans [1,2]. These compounds can be used in many industrial applications such as light sensitive sunglasses [3,4], molecular electronics [5], optical memories [6-9]. On the other hand, many natural naphtho- and benzopyrans exhibit pharmacological applications [10,11] such as anti-HIV [12], antihypertensive [13] and anti-ischaemic [14] properties.

But a very few studies concern the design of new photochromic systems. Presence of phenyl in position 6 of 6,6a-dihydro-[1]benzopyrano-[3,4-b][1]benzopyran derivatives is needed for the use of photochromic properties of these systems [2].

Similar benzopyran have been prepared as key intermediates in synthetic routes leading to rotenoids $[15,16]$. However, synthesis of compounds with phenyl group in position 6 is not described. This paper reports synthesis of the first 6-substitued 6,6a-dihydro-[1]benzopyrano-[3,4-b][1]benzopyran using, with some modifications, the approach proposed by Pastine and Sames [17]. This process is based on two key cyclisation steps involving respectively a platinum-catalysed 6-endohydroarylation of alkynones and a oxo-Michael addition.

\section{RESULTS AND DiscuSSION}

The eight steps allowing to the target benzopyran are reported on Figure 1.

The propargylic ether $\mathbf{1}$, needed for the synthesis of the compounds 2 , was prepared using the Mitsonobu reaction [18] between the $m$-methoxyphenol and the phenylprop-3-ynol. (3-methoxy-1-phenoxy)-1phénylprop-2-yne 1 was obtained with a yield of 31\%. Balasubramanian and al described similar yields (35 to $55 \%$ ) in their work about the synthesis of aromatic propargylic ethers [19-21]. The low yield observed is mainly due to the conversion of propynol to the corresponding allene.

The reaction of the acetylure 1 with $o$-methoxy benzaldehyde gave the expected 1-(2-methoxyphenyl)4-(3-methoxyphenoxy)-4-phenylbut-2-yn-1-ol 2 with $77 \%$ yield. Oxidation of 2 into ketone was realized using $\mathrm{CrO}_{3}$ with catalytic amount of $\mathrm{H}_{2} \mathrm{SO}_{4}$ [22] and gave 1-(2-methoxyphenyl)-4-(3-methoxyphenoxy)-4-phenylbut2-yn-1-one 3 with $68 \%$ yield.

The key cyclisation step leading to chromene was realized using $\mathrm{PtCl}_{4}$ as catalyst and gave 4-(2methoxyphenylcarbonyl)-7-methoxy-1-phenylbenzopyran 4 (38\%). When $\mathrm{PtCl}_{2}$ is used instead of $\mathrm{PtCl}_{4}$, the yield decreases (29\%). This result is conform to others described in the literature [23]. Howover, Federov and al showed that the intramolecular hydroarylation can be obtained in higher yield (60-91\%) using catalytic amount of $\mathrm{Pd}(\mathrm{OAc})_{2}$ [24].

The following demethylation step is not very efficient due to the presence of the second methoxy group [25]. 4-(2-hydroxyphenylcarbonyl)-7-methoxy-1-phenylbenzopyran $\mathbf{5}$ was isolated after purification with $23 \%$ yield. The use of another electrodonor group insensitive to the action of $\mathrm{BCl}_{3}$ in place of methoxy group of position 3 will allow the reaction to give better yields [24]. 
The second key-cyclisation reaction is realized through an oxo-Michael addition in mild basic conditions (potassium acetate in refluxing ethanol). Probability that the mechanisms proposed on the literature $26,27]$ take place is very low because of the formation of tense cycles. Before dehydration with catalytic amount of PTSA refluxing in the toluene, the crude residue was first treated with $\mathrm{LiAlH}_{4}$ in the anhydrous THF. Finally, 3-methoxy-6-phenyl-6,6a-dihydro-[1]benzopyrano-[3,4-b][1]benzopyran 6 was isolated with 30\% yield.

\section{EXPERIMENTAL}

The reactions were monitored by thin-layer chromatography on aluminium plates precoated with Merck silica gel 60 F254 (0.25 mm). Column chromatography (CC) was performed on silica gel 60 (230 - 400 mesh). The new compounds were determined to be $>95 \%$ pure by ${ }^{1} \mathrm{H}$ NMR spectroscopy and gas chromatography (GC). Mass spectrometer coupling with GC were made with a HP 5973 apparatus with HP-5 $5 \%$ Phenylmethylsiloxan column using helium as a vector gas. The ${ }^{1} \mathrm{H}$ and ${ }^{13} \mathrm{C} \mathrm{nmr}$ spectra were recorded at $300 \mathrm{~K}$ in $\mathrm{CDCl}_{3}$ using a Bruker AC250 spectrometer (at 250 and $62.5 \mathrm{MHz}$ ). Tetramethylsilan (TMS) was used as an internal standard. Chemical shifts $(\delta)$ are reported in ppm and coupling constants $(\mathrm{J})$ in $\mathrm{Hz}$. UV-Vis spectra were recorded on a Beckman DU 7500 spectrophotometer. I.R spectra were obtained on a Perkin - Elmer 297 spectrometer using $\mathrm{NaCl}$ disks (wavelength in $\mathrm{cm}^{-1}$ ). Elemental analysis were made by "Spectropole" (AixMarseille III University-France). Melting points ( $\mathrm{Mp}$ in ${ }^{\circ} \mathrm{C}$ ) were measured with Electrothermal IA 9100.

If not indicated, the experimental procedures for the synthesis of the different intermediates are those described by Pastine and Sames [20].

(3-methoxy-1-phenoxy)-1-phénylprop-2-yne (1). $3.6 \mathrm{~mL}(23 \mathrm{mmol}$; $\mathrm{d}=1.1)$ of diethyl azodicarboxlylate (DEAD) was slowly added, under argon, to a mixture of $3 \mathrm{~g}(23 \mathrm{mmol})$ of 1-phenylprop-2-yn-1-ol, $2.86 \mathrm{~g}$ (23 mmol) of 2-methoxyphenol and $6 \mathrm{~g}(23 \mathrm{mmol})$ of triphenylphosphin in $300 \mathrm{~mL}$ of dry dichloromethane. After stirring at room temperature for $22 \mathrm{~h}$, the mixture was concentrated under reduce pressure. The crude residue was purified by Column Chromatography (97\% $n$-hexane and 3\% diethylether) and gave yellow oil, $1.7 \mathrm{~g}$ (31\%). ir: Csp 3291. ${ }^{1} \mathrm{H}$ nmr (deuteriochloroform): $\delta 2.64(\mathrm{~s}, 1 \mathrm{H}), 3.70(\mathrm{~s}, 3 \mathrm{H}), 5.77(\mathrm{~s}, 1 \mathrm{H}), 6.49(\mathrm{~s}, 1 \mathrm{H}), 6.52-$ $6.60(\mathrm{~m}, 2 \mathrm{H}), 7.16(\mathrm{~m}, 1 \mathrm{H}), 7.30-7.35(\mathrm{~m}, 3 \mathrm{H}), 7.53-7.56(\mathrm{~d}, 2 \mathrm{H}, \mathrm{J}=6.5 \mathrm{~Hz}) .{ }^{13} \mathrm{C} \mathrm{nmr}$ (deuteriochloroform): $\delta$ 54.25, 68.77, 75.42, 79.86, 101.43, 106.48, 106.95, 126.24, 127.70, 127.84, 128.80, 136.28, 157.48, 159.66. Anal. Calcd. For $\mathrm{C}_{16} \mathrm{H}_{14} \mathrm{O}_{2}$ : C, 80.65; H, 5.92; O, 13.43. Found: C, 80.69; H, 5.91; O, 13.4.

1-(2-methoxyphenyl)-4-(3-methoxyphenoxy)-4-phenylbut-2-yn-1-ol (2). Synthesis gave Brown oil, $1.73 \mathrm{~g}$ (77\%). ir: $\mathrm{OH} 3419 .{ }^{1} \mathrm{H} \mathrm{nmr}$ (deuteriochloroform) : $\delta 3.13(\mathrm{~s}, 1 \mathrm{H}), 3.57(\mathrm{~s}, 3 \mathrm{H}), 3.61(\mathrm{~s}, 3 \mathrm{H}), 5.60(\mathrm{~s}, 1 \mathrm{H}), 5.77$ $(\mathrm{s}, 1 \mathrm{H}), 6.45(\mathrm{~d}, 1 \mathrm{H}, \mathrm{J}=8,2 \mathrm{~Hz}), 6.53-6.62(\mathrm{~m}, 2 \mathrm{H}), 6.69-6.79(\mathrm{~m}, 2 \mathrm{H}), 7.04-7.14(\mathrm{~m}, 2 \mathrm{H}),, 7.23-7.32(\mathrm{~m}, 4 \mathrm{H})$, $7.48(\mathrm{~d}, \mathrm{~J}=6.5 \mathrm{~Hz}, 2 \mathrm{H}) .{ }^{13} \mathrm{C} \mathrm{nmr}$ (deuteriochloroform): $\delta$ 54.12, 54.28, 60.07, 69.14, 81.80, 87.14, 101.46, $106.43,107.20,109.64,119.65,126.35,126.84,127.24,127.54,127.62,128.69,136.58,155.62,157.59$, 159.55. Anal. Calcd for $\mathrm{C}_{24} \mathrm{H}_{22} \mathrm{O}_{4}$ : C, 76.99; H, 5.92; O, 17.09. Found: C, 76.97; H, 5.92; O, 17.11.

1-(2-methoxyphenyl)-4-(3-methoxyphenoxy)-4-phenylbut-2-yn-1-one (3). $85.7 \mathrm{~g}(9 \mathrm{mmol}) \quad$ of $\mathrm{CrO}_{3}$ was slowly added to a solution of $1.7 \mathrm{~g}(4.5 \mathrm{mmol})$ of 1-(2-methoxyphenyl)-4-(3-methoxyphenoxy)-4-phenylbut-2yn-1-ol (2) in $30 \mathrm{~mL}$ of dry DMF. Then 5 drops of concentrated sulphuric acid was added to the mixture. After stirring at room temperature for $40 \mathrm{~min}$., water $(40 \mathrm{~mL})$ and diethyelether $(60 \mathrm{~mL})$ was added and the aqueous phase was extracted with diethylether. The combined organic layers was washed with satured solution of sodium chloride, dried in magnesium sulphate and concentrated under reduce pressure. The residue was purified by column chromatography ( $85 \% \mathrm{n}$-hexane and $15 \%$ diethylether). Reaction gave yellow oil, $1.15 \mathrm{~g}(68 \%)$. ${ }^{1} \mathrm{H} \mathrm{nmr}$ (deuteriochloroform): $\delta 3.61(\mathrm{~s}, 3 \mathrm{H}), 3.66(\mathrm{~s}, 3 \mathrm{H}), 5.94(\mathrm{~s}, 1 \mathrm{H}), 6.49(\mathrm{~d}, 1 \mathrm{H}, \mathrm{J}=8.2 \mathrm{~Hz}), 6.57-6.65(\mathrm{~m}$, $2 \mathrm{H}), 6.79-6.82(\mathrm{~m}, 2 \mathrm{H}), 7.11(\mathrm{~m}, 1 \mathrm{H}), 7.28-7.33(\mathrm{~m}, 4 \mathrm{H}), 7.53(\mathrm{~m}, 2 \mathrm{H}), 7.76(\mathrm{~d}, 1 \mathrm{H}, \mathrm{J}=7.7 \mathrm{~Hz}) .{ }^{13} \mathrm{C} \mathrm{nmr}$ (deuteriochloroform): $\delta 56.94,57.16,71.76,89.26,90.40,104.19,109.50,109.67,113.67,121.88,127.60$, 129.12, 130.50, 130.79, 131.63, 134.22, 137.04, 138.01, 160.06, 161.60, 162.43, $177.51(\mathrm{C}=\mathrm{O})$. Anal. Calcd. for $\mathrm{C}_{24} \mathrm{H}_{20} \mathrm{O}_{4}$ : C, 77.40; H, 5.41; O, 17.18). Found: C, 77.41; H, 5.38; O, 17.21.

4-(2-methoxyphenylcarbonyl)-7-methoxy-1-phenylbenzopyran (4). After purification by column chromatography (90\% n-hexane and $10 \%$ diethylether) yellow oil was obtained, $0.43 \mathrm{~g}(38 \%)$. ${ }^{1} \mathrm{H} \mathrm{nmr}$ (deuteriochloroform): $\delta 3.60(\mathrm{~s}, 3 \mathrm{H}), 3.66(\mathrm{~s}, 3 \mathrm{H}), 5.78(\mathrm{~d}, 1 \mathrm{H}, \mathrm{J}=3.7 \mathrm{~Hz}), 6.00(\mathrm{~d}, 1 \mathrm{H}, \mathrm{J}=3.7 \mathrm{~Hz}), 6.37(\mathrm{~m}$, $2 \mathrm{H}), 6.78-6.93(\mathrm{~m}, 2 \mathrm{H}), 7.23-7.42(\mathrm{~m}, 7 \mathrm{H}), 7.56(\mathrm{~d}, 1 \mathrm{H}, \mathrm{J}=8.0 \mathrm{~Hz}) .{ }^{13} \mathrm{C} \mathrm{nmr}$ (deuteriochloroform): $\delta 54.25$, 54.47, 75.11, 101.14, 106.26, 110.49, 111.32, 119.47, 126.04, 126.20, 127.70, 128.38, 129.26, 129.48, 131.84, 134.14, 138.26, 153.48, 156.89, 160.04, $195.02(\mathrm{C}=\mathrm{O})$. Anal. Calcd. For $\mathrm{C}_{24} \mathrm{H}_{20} \mathrm{O}_{4}$ : C, 77.40; H, 5.41; O, 17.18. Found: C, 77.39; H, 5.38; O: 17.23.

4-(2-hydroxyphenylcarbonyl)-7-methoxy-1-phenylbenzopyran (5). $1.3 \mathrm{~mL}(1.3 \mathrm{mmol})$ of a $1 \mathrm{M}$ solution of $\mathrm{BCl}_{3}$ was slowly added, under argon at $-78^{\circ} \mathrm{C}$, to a solution of $0.4 \mathrm{~g}$ (1.1 mmol) of 4-(2- 
methoxyphenylcarbonyl)-7-methoxy-1-phenylbenzopyran (4) in $30 \mathrm{~mL}$ of dry dichloromethane. After stirring for $1 \mathrm{~h}$, the mixture was quenched with saturated solution of ammonium chloride $(20 \mathrm{~mL})$, extracted with ethylacetate and the combined organic layers dried in magnesium sulphate and concentrated under reduce pressure after filtration. The residue was purified by column chromatography $(90 \% n$-hexane and $10 \%$ diethylether). Reaction gave yellow oil, $0.091 \mathrm{~g}(23 \%) .{ }^{1} \mathrm{H} \mathrm{nmr}$ (deuteriochloroform): $\delta 3.69(\mathrm{~s}, 3 \mathrm{H}), 5.85(\mathrm{~d}$, $1 \mathrm{H}, \mathrm{J}=3.5 \mathrm{~Hz}), 5.93(\mathrm{~d}, 1 \mathrm{H}, \mathrm{J}=3.5 \mathrm{~Hz}), 6.40(\mathrm{~m}, 2 \mathrm{H}), 6.76(\mathrm{~m}, 1 \mathrm{H}), 6.98(\mathrm{~m}, 2 \mathrm{H}), 7.31-7.43(\mathrm{~m}, 6 \mathrm{H}), 7.65(\mathrm{~d}$, $1 \mathrm{H}, \mathrm{J}=7.7 \mathrm{~Hz}, 1 \mathrm{H}), 11.96(\mathrm{~s}, 1 \mathrm{H}, \mathrm{OH}) .{ }^{13} \mathrm{C} \mathrm{nmr}$ (deuteriochloroform): $\delta 54.34,75.01,100.92,108.16,112.47$, $110.82,119.67,123.42,127.87,128.24,128.86,128.13,128.95,129.21,132.14,133.18,138.31,154.47$, 157.74, 160.09, $198.67(\mathrm{C}=\mathrm{O})$. Anal. Calcd. For $\mathrm{C}_{23} \mathrm{H}_{18} \mathrm{O}_{4}$ : C, 77.08; H, 5.06; O, 17.86. Found: C, 77.07; H, $5.09 ; \mathrm{O}, 17.84$.

3-methoxy-6-phenyl-6,6a-dihydro-[1]benzopyrano-[3,4-b][1]benzopyran (6). A solution of $0.075 \mathrm{~g}(0.21$ $\mathrm{mmol}$ ) of 4-(2-hydroxyphenylcarbonyl)-7-methoxy-1-phenylbenzopyran (5) in $10 \mathrm{~mL}$ of ethanol was saturated with potassium acetate. The mixture was heated under reflux for $1 \mathrm{~h}$. After cooling at room temperature ethyl acetate $(20 \mathrm{~mL})$ and water $(10 \mathrm{~mL})$ was added. The aqueous layer was extracted with ethyl acetate and the combined organic layers washed with saturated solution of sodium chloride, dried in magnesium sulphate and concentrated under reduce pressure. A solution of the crude residue in $10 \mathrm{~mL}$ of dry THF was added dropwise, under argon, to a suspension of $\mathrm{LiALH}_{4}$ in $5 \mathrm{~mL}$ of dry THF. After stirring at room temperature for $10 \mathrm{~min}$., the solution was quenched with saturated solution of ammonium chloride $(10 \mathrm{~mL})$, extracted with diethylether and the combined organic layers dried in magnesium sulphate and concentrated under reduce pressure. The residue was dissolved in $20 \mathrm{~mL}$ of toluene and heated under reflux for $15 \mathrm{~min}$. in the presence of catalytic quantity of para-toluensulfonic acid. $5 \mathrm{H}_{2} \mathrm{O}$. After concentrated under reduced pressure, the crude residue was purified by column chromatography (97\% $n$-hexane and 3\% diethyl ether). The target compound (6) was an yellow-green oil, $0.025 \mathrm{~g} \mathrm{(30 \% ).}{ }^{1} \mathrm{H} \mathrm{nmr}$ (deuteriochloroform): $\delta 3.72(\mathrm{~s}, 3 \mathrm{H}), 5.10(\mathrm{~d}, 1 \mathrm{H}, \mathrm{J}=10.0 \mathrm{~Hz}), 5.22(\mathrm{dd}, 1 \mathrm{H}, \mathrm{J}=$ 10.0, $2.2 \mathrm{~Hz}), 6.42$ (d, 1H, J = 2.5 Hz), $6.57(\mathrm{~m}, 2 \mathrm{H}), 6.70(\mathrm{~m}, 1 \mathrm{H}), 6.82(\mathrm{~d}, 1 \mathrm{H}, \mathrm{J}=7.2 \mathrm{~Hz}), 6.91-7.01(\mathrm{~m}, 2 \mathrm{H})$, $7.12-7.19(\mathrm{~m}, 4 \mathrm{H}), 7.38-7.48(\mathrm{~m}, 2 \mathrm{H}) .{ }^{13} \mathrm{C} \mathrm{nmr}$ (deuteriochloroform): $\delta 54.42,77.85,79.07,100.57,109.26$, $111.30,114.67,120.95,122.46,123.68,124.26,125.44,126.76,127.19,128.00,129.47,133.48,136.67$, 151.57, 155.07, 160.00. Anal. Calcd. For $\mathrm{C}_{23} \mathrm{H}_{18} \mathrm{O}_{3}$ : C, 80.68; H, 5.30; O, 14.02. Found: C, 80.70; H, 5.30; O: 14.

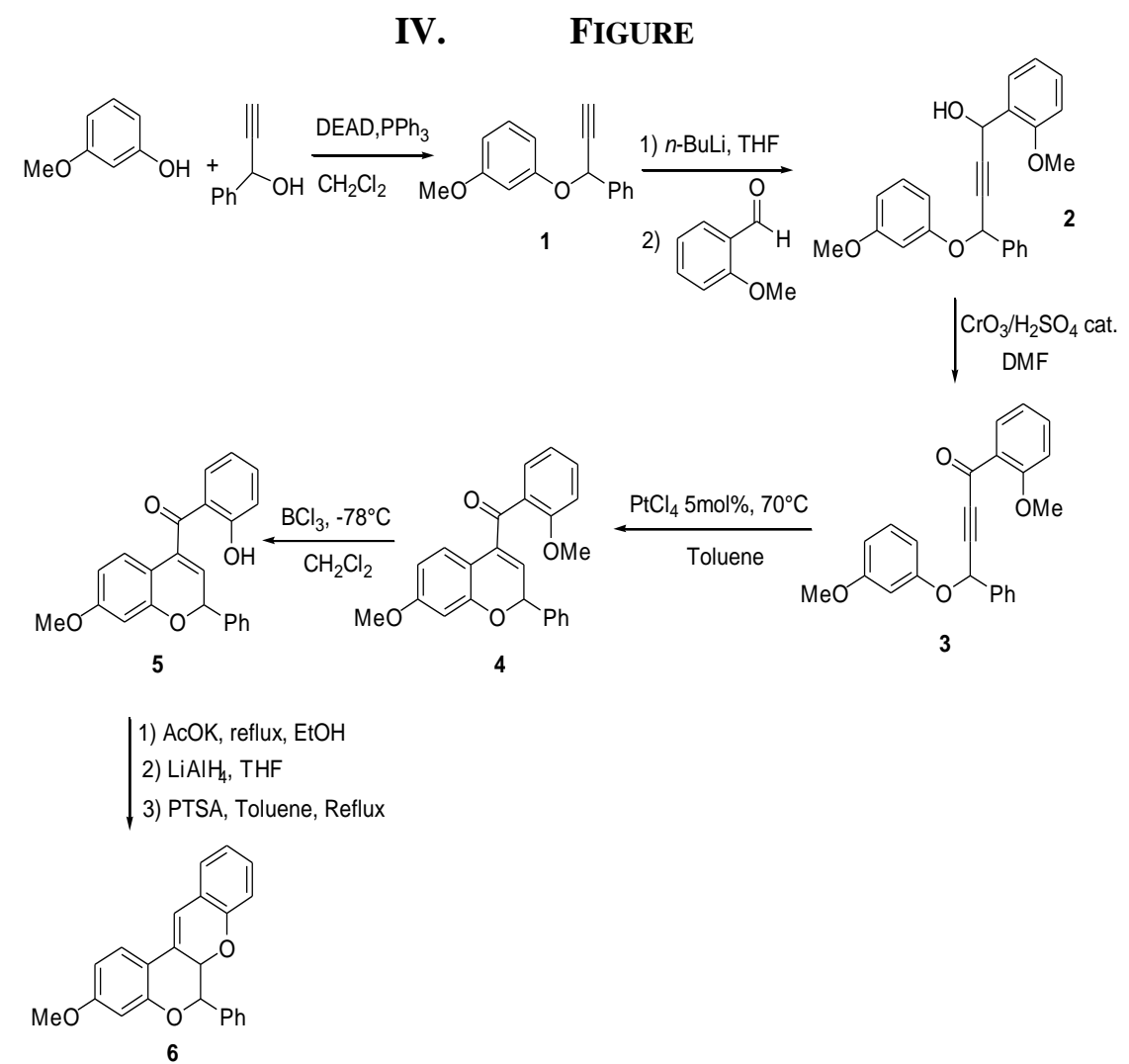

Figure 1. Synthetic pathway to the 3-methoxy-6-phenyl-6,6a-dihydro-[1]benzopyrano-[3,4-b][1]benzopyran (6). 


\section{CONCLUSION}

In summary, we have reported an efficient method for the synthesis of the 6-substitued 6,6a-dihydro[1]benzopyrano-[3,4-b][1]benzopyran. Presence of phenyl group in position 6 is indispensable to stabilize the open form of this family of compounds for their potential photochromic applications [28].

\section{Acknowledgments}

We would like to thank Dr. G. Buntinx and O. Poizat (LASIR UMR 8516-University Lille 1, France) for the preliminary photochemical experiment performed on the 3-methoxy-6-phenyl-6,6a-dihydro[1]benzopyrano-[3,4-b][1]benzopyran (6).

We dedicated this paper to the memory of the Professor Pierre Brun.

\section{References}

[1] Dürr, H.; Bouas-Laurent, H. In Photochromism : Molecules and Systems; Eds.; Elsevier: Amsterdam, 1990.

[2] Van Gemert, B. In Organic photochromic and thermochromic compounds. Main photochromic families; Crano J.C.; Guglielmetti, R.; Ed.; Plenum Press: New York, 1999; Vol. 1, 111.

[3] Mc Ardle, G. In Applied Photochromic Polymers Systems; Ed.; Blackie: London, 1992.

[4] Hepworth, J.D.; Heron, B.M. In Functional Dyes, Sung-Hoon Kim, Ed.; Elsevier: Amsterdam $2006 ; 85$.

[5] Delaire, J.A.; Nakatami, K. Chem. Rev. 2000, 100, 1817.

[6] Irié, M. Chem. Rev. 2000, 100, 1685.

[7] Berkovic, G.; Krongauz, V.; Weiss, V. Chem. Rev. 2000, 1741.

[8] Kawata, S.; Kawata, Y. Chem. Rev. 2000, 100, 1777.

[9] Ferringa, B.L.; Jager, W.F.; Delange, G.B. Tetrahedron 1993, 49, 8267.

[10] Cassidy, F.; Evans, J.M.; Hadley, M.S.; Haladij, A.H.; Leach, P.E.; Stemp, G. J. Med. Chem. 1992, 35, 1623.

[11] Banskota, A.H.; Tezuka, Y.; Prasain, J.K.; Matsuhige, I.S.; Kadota, S. J. Nat. Prod. 1998, 61, 896.

[12] McKee, T.C.; Fuller, R.W.; Covington, C.D.; Cardellina, J.H.; Gulakowski, R.J.; Krepps, B.L.; McMahon, J.B.; Boyd, M.R. J. Nat. Prod. 1996, 59, 754

[13] Ashwood, V.A.; Cassidy, F.; Codwell, M.C.; Evans, J.M.; Hamilton, T.C.; Howlett, D.R.; Smith, D.M.; Stemp, G. .J. Med. Chem. 1990, 33, 2667.

[14] Atwal, K.S.; Grover, G.J.; Ferrara, F.N.; Ahmed, S.Z.; Sleph, P.G.; Dzwonczyk, S.; Normandin, D.E. J. Med. Chem. 1995, 35, 1966.

[15] Asiah Ahmad-Junan, S.; Whiting, D.A. J. Chem. Soc., Chem. Commun. 1988, 17, 1160.

[16] Asiah Ahmad-Junan, S.; Amos, P.C.; Whiting, D.A. J. Chem. Soc., Faraday Trans 1 1992, 5, 539.

[17] Pastine, S.J.; Sames, D. Org. Lett. 2003, 22, 4053.

[18] Mitsunobu, O. Synthesis 1981, 1 .

[19] Subramanian, R.S.; Balasubramanian, K.K. Tetrahedron Lett. 1988, 29, 6797.

[20] Subramanian, R.S.; Balasubramanian, K.K. Synth. Commun. 1989, 19, 1255.

[21] Subramanian, R.S.; Balasubramanian, K.K. Tetrahedron Lett. 1989, 30, 2297.

[22] Snatzke, G. Chem. Ber. 1961, 94, 729.

[23] Mamane, V; Hannen, P; Fürstner, A. Chem. Eur. J. 2004, 10, 4556

[24] Fedorov, A.Y.; Nyuchev, A.V.; Beletskaya, I.P. Chem. Heterocycl. Comp. 2012, 48, 166.

[25] Bellur, E; Langer, P. J. Org. Chem. 2005, 70, 7686.

[26] Maiti, S.; Kalyan Panja, S.; Bandyopadhyay, C. Tetrahedron. 2010, 66, 7625.

[27] Ghabraie, E.; Bararjanian, M.; Balalaie, S.; Rominger, F.; Bijanzadeh, H.R. Helv. Chim. Acta 2011, $94,1440$.

[28] Abogo Mebale, AJ; Maggiani, A.; Ondo Ndong, R.; Buntinx, G.; Ondo, J.A.; Gnanga, H.; Samat, A. Chemical and Process Engineering Research. 2013, 8, 1. 